\title{
Inhibitory effects of plasmid small interfering RNA targeting signal transducer and activator of transcription-3 in C6 glioma cells
}

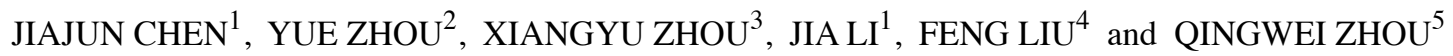 \\ ${ }^{1}$ Department of Neurology, China-Japan Union Hospital of Jilin University, Changchun, Jilin 130033; \\ ${ }^{2}$ Department of Biotherapeutics, College of Pharmacy, Nanjing University of Chinese Medicine, Nanjing, Jiangsu 210000; \\ ${ }^{3}$ Department of Hepatobiliary Surgery, The Second Hospital of Jilin University, Changchun, Jilin 130041; \\ ${ }^{4}$ Department of Nephrology, China-Japan Union Hospital of Jilin University, Changchun, Jilin 130033; \\ ${ }^{5}$ Biomedical Engineering Unit, Institute of Frontier Medical Sciences of Jilin University, \\ Changchun, Jilin 130021, P.R. China
}

Received April 2, 2018; Accepted November 5, 2018

DOI: $10.3892 / \mathrm{mmr} .2019 .10459$

\begin{abstract}
The current study investigated the effect of plasmid small interfering RNA (psiRNA)-mediated silencing of signal transducer and activator of transcription-3 (STAT3) on the invasion, apoptosis, and expression levels of cyclin D1, caspase-3 and B-cell lymphoma-2 (Bcl-2) in C6 glioma cells. Cell invasion was determined using a Transwell assay, while the apoptosis rate and cell cycle distribution of cells were assessed using Annexin V-FITC/PI double staining. The expression levels of cyclin D1, caspase-3 and Bcl-2 proteins were measured by western blotting. Transfection with psiRNA-STAT3 was observed to significantly decrease the number of transmembrane cells compared with the control groups $(\mathrm{P}<0.05)$. In addition, the proportion of cells at G0/G1 phase was increased in the psiRNA-STAT3 group compared with the controls. Western blotting indicated that psiRNA-STAT3 decreased the expression of cyclin D1, caspase-3 and Bcl-2 proteins. Taken together, psiRNA-STAT3 inhibited the migration and invasive abilities, and induced the apoptosis of C6 glioma cells, possibly through regulation of the expression of cyclin D1, caspase-3 and $\mathrm{Bcl}-2$ proteins.
\end{abstract}

Correspondence to: Dr Feng Liu, Department of Nephrology, China-Japan Union Hospital of Jilin University, 126 Xiantai Street, Changchun, Jilin 130033, P.R. China

E-mail: jdliufeng@163.com

Dr Qingwei Zhou, Biomedical Engineering Unit, Institute of Frontier Medical Sciences of Jilin University, 1163 Xinmin Street, Changchun, Jilin 130021, P.R. China

E-mail: zhouqw@jlu.edu.cn

Key words: plasmid small interfering RNA, signal transducer and activator of transcription-3, glioma cells, apoptosis, protein expression, cell cycle

\section{Introduction}

Gliomas are the most common malignant intracranial tumors of the human central nervous system $(1,2)$, and their occurrence and development involve multi-step and multi-factorial processes. Traditional treatments, such as surgery, radiotherapy and chemotherapy, have low cure rates and high tumor recurrence rates, and are associated with poor prognosis (3). Various newly developed therapies, including genetic immunotherapy, microsurgery (4), new radiotherapy and chemotherapy strategies (5), anti-invasion therapy (6) and anti-angiogenesis therapy (7), have recently led to great progress in the treatment of gliomas and other malignant tumors $(8,9)$. However, due to the characteristics of infiltrative growth (10), increased drug tolerance (11) and high invasiveness (12) of gliomas, these treatments have not achieved satisfactory results in all clinical trials (13), and patient prognosis has not been significantly improved.

Current novel antitumor treatment strategies aim to simultaneously target different aspects of the tumorigenesis mechanism to achieve better efficacy. Specific targeting of signal transducer and activator of transcription-3 (STAT3) signaling transduction has potential value for the treatment of malignant glioma (14).

In our previous study, it was demonstrated that transfection with STAT3-specific silencing RNA, namely plasmid small interfering RNA (psiRNA)-STAT3, induced apoptosis of glioma cells through the endogenous caspase pathway mediated by the mitochondrial membrane potential, thus inhibiting the growth of tumor cells (15). The present study aimed to further investigate the underlying mechanisms of the effects of psiRNA-STAT3 on C6 glioma cells in order to provide a theoretical basis for clinical research into glioma treatment.

\section{Materials and methods}

Cells and reagents. Rat C6 glioma cells were purchased from the Shanghai Institute of Cell Biology (Chinese Academy of Sciences, Shanghai, China). Opti-minimum essential medium 
(MEM; Gibco) and Lipofectamine ${ }^{\circledR} 2000$ reagent (Invitrogen; cat. no. 11668-019) were purchased from Thermo Fisher Scientific, Inc. (Waltham, MA, USA). psiRNA-STAT3 and scramble psiRNA were constructed at the Department of Pathophysiology of the Institute of Basic Medicine of Jilin University (Changchun, China). The si-STAT3 sequence was GCAGCAGCTGAACAACAT (spanning nucleotides 2,144-2,162; GenBank accession no. NM003150). Transwell chambers were obtained from Corning, Inc. (Corning, NY, USA). Pounceau S staining solution, Triton X-100, 5X sodium dodecyl sulfate (SDS) loading buffer and the Annexin V-FITC cell apoptosis detection kit (cat. no. GK3603-50T) were purchased from Beijing Dingguo Changsheng Biotechnology Co., Ltd. (Beijing, China). Ribonuclease (cat. no. ST577), BeyoECL Plus (cat. no. P0018S) was purchased from Beyotime Institute of Biotechnology (Shanghai, China). Rabbit anti-cyclin D1 (cat. no. BA0770), rabbit anti-B-cell lymphoma-2 (Bcl-2; cat. no. A00040-1), rabbit anti-caspase-3 (cat. no. BA2142), horseradish peroxidase-conjugated goat anti-rabbit IgG (cat. no. BA1055) and anti- $\beta$-actin (cat. no. BM0626) antibodies, and propidium iodide were obtained from Wuhan Boster Biological Technology, Ltd. (Wuhan, China). Radioimmunoprecipitation assay (RIPA) buffer was purchased from Beyotime Institute of Biotechnology.

Instruments. The ECLIPSE 80i inverted microscope was obtained from Nikon Corporation (Tokyo, Japan), and the Guava easyCyte 6HT-2L flow cytometer powered by GuavaSoft 1.0 was from Merck KGaA (Darmstadt, Germany). The WH-600-LCD electrophoresis apparatus was purchased from Shanghai Qite Analytical Instrument Co., Ltd. (Shanghai, China). The JY-ZY5 metastatic electrophoresis bath was from Beijing JunYi DongFang Electrophoresis Co., Ltd. (Beijing, China). The Molecular Imager Gel Doc XR/XRS system was purchased from Bio-Rad Laboratories, Inc. (Hercules, CA, USA), and the MCO-15AC $\mathrm{CO}_{2}$ incubator was from Sanyo Electric Co., Ltd. (Osaka, Japan).

Treatment groups and transfection. Opti-MEM medium (225 $\mu \mathrm{l} /$ tube) was added to four Eppendorf tubes marked as tubes 1-4, and then the following solutions were added to each tube: Tube 1, $4.4 \mu 1$ psiRNA-STAT3 pharmaceutical solution; tube 2, $4.4 \mu$ 1 Scramble plasmid solution; and tubes 3 and $4,11 \mu \mathrm{l}$ Lipofectamine ${ }^{\circledR}$ 2000. Following incubation for 5 min, tube 1 was mixed with 3 and tube 2 was mixed with 4 , and all tubes were incubated for a further $20 \mathrm{~min}$. Cultured C6 glioma cells were divided into the Mock, Scramble and psiRNA-STAT3 groups. A total of $514 \mu \mathrm{l}$ of the appropriate plasmid solution was added to each well in the Scramble and psiRNA-STAT3 groups, while the Mock group was not treated with plasmid. Cells were transfected according to the manufacturer's protocol for Lipofectamine ${ }^{\circledR} 2000$. The successful transfection of psiRNA-STAT3 C6 glioma cells was verified in our previous study, which resulted in mitochondrial membrane potential reduction and subsequent apoptosis (16). In the present study, the cell cycle and associated protein expression was examined.

Determination of cellular invasion ability by Transwell assay. Precooled serum-free DMEM was mixed with liquid Matrigel matrix at a ratio of $1: 5$, and $40 \mu \mathrm{l}$ of this mixture was added to the upper part of Transwell chambers and incubated at $37^{\circ} \mathrm{C}$ for $5 \mathrm{~h}$. Serum-free DMEM (70 $\mu \mathrm{l})$ was added to the upper part of Transwell chambers, followed by addition of $200 \mu \mathrm{l}$ cell suspension $\left(4 \times 10^{5} / \mathrm{ml}\right)$ in each well of the Transwell chambers. A total of $500 \mu 1$ medium containing $30 \%$ calf serum was added to the lower chambers. All groups were incubated at $37^{\circ} \mathrm{C}$ with $5 \% \mathrm{CO}_{2}$ for $8 \mathrm{~h}$, and the culture solution in the upper part of the Transwell chambers was then discarded. Cells that had invaded the semipermeable membrane and attached to the lower chamber were fixed with $10 \%$ formaldehyde for $20 \mathrm{~min}$ at room temperature, stained with crystal violet for $15 \mathrm{~min}$ and then examined by inverted microscopy. Three random fields-of-view were selected, and the mean number of invading cells was calculated for each experimental group.

Determination of cell cycle distribution by flow cytometry. C6 glioma cells in the logarithmic phase were detached by digestion with $0.25 \%$ trypsin and centrifuged at $377 \mathrm{x}$ g for $5 \mathrm{~min}$ at room temperature. The supernatant was discarded, and the cells were seeded at $1 \times 10^{6}$ cells/well in 6-well plates and incubated at $37^{\circ} \mathrm{C}$ with $5 \% \mathrm{CO}_{2}$ for $8 \mathrm{~h}$. The cells were harvested and mixed with $80 \mu 1$ each of ribonuclease, propidium iodide and Triton X-100. Following incubation at room temperature for $20 \mathrm{~min}$, the cell cycle distribution was analyzed by flow cytometry.

Detection of apoptosis by flow cytometry. C6 glioma cells in the logarithmic phase were cultured and harvested as described earlier. Cells were then mixed gently with $195 \mu \mathrm{l}$ Annexin V-FITC binding buffer, $5 \mu \mathrm{l}$ Annexin V-FITC and $10 \mu 1$ propidium iodide staining solution. Following incubation for $10 \mathrm{~min}$ at room temperature, cells were detected by flow cytometry to analyze the fractions of dead (quadrant P1), non-apoptotic healthy (quadrant P2), late apoptotic (quadrant P3) and early apoptotic (quadrant P4) cells.

Detection of cyclin D1, caspase-3 and Bcl-2 expression by western blotting. C6 glioma cells in the logarithmic phase were detached by digestion with $0.25 \%$ trypsin and centrifuged at room temperature and $377 \mathrm{x}$ g for $5 \mathrm{~min}$. The supernatant was discarded, and the cells were seeded at $1 \times 10^{6}$ cells/well in 6-well plates and incubated at $37^{\circ} \mathrm{C}$ with $5 \% \mathrm{CO}_{2}$ for $8 \mathrm{~h}$. Next, the culture solution was discarded, RIPA lysis buffer was added to the cells, and the solution was centrifuged at $4^{\circ} \mathrm{C}$ for $30 \mathrm{~min}$ at $15,535 \mathrm{x}$ g. Protein concentration was determined with a bicinchoninic acid protein assay kit. Protein (50 $\mu \mathrm{g} / \mathrm{lane}$ ) was separated by $10 \%$ SDS-PAGE and electrotransferred to a cellulose membrane under transfer conditions of $200 \mathrm{~mA}$ for $90 \mathrm{~min}$, followed by $0.1 \%$ Pounceau S staining for $10 \mathrm{~min}$ at room temperature to reversibly detect the protein bands. The stain was removed with TBST. Subsequent to blocking at $4^{\circ} \mathrm{C}$ for $1 \mathrm{~h}$ in $5 \%$ non-fat milk in PBS buffer containing $0.1 \%$ Tween-20, the membranes were incubated with primary antibodies against cyclin D1, caspase-3, Bcl-2 and $\beta$-actin (1:300) at $4^{\circ} \mathrm{C}$ overnight, followed by incubation with secondary antibodies $(1: 3,000)$ at room temperature for $1 \mathrm{~h}$. Protein expression was detected by enhanced chemiluminescence reagent (Beyotime Institute of Biotechnology). Band density was analyzed with GelCapture software version 7.4.0 (DNR Bio-Imaging Systems, Ltd., Neve Yamin, Israel). 


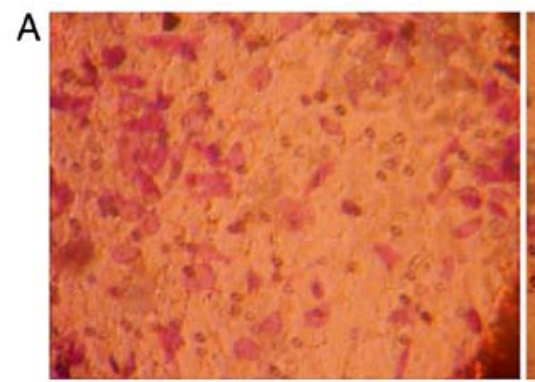

Mock group $(\times 20)$

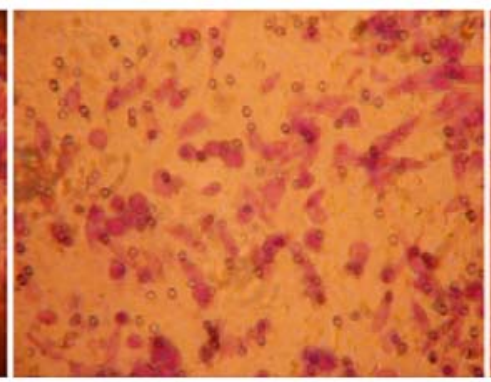

Scramble group $(\times 20)$

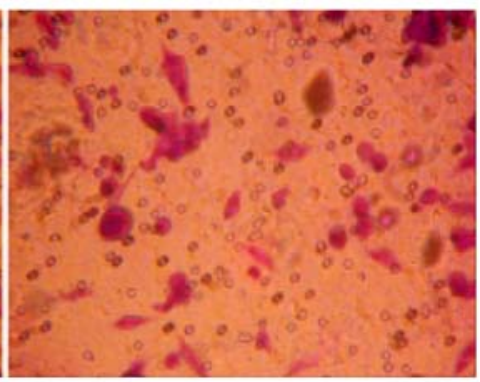

PsiRNA-STAT3 group $(\times 20)$

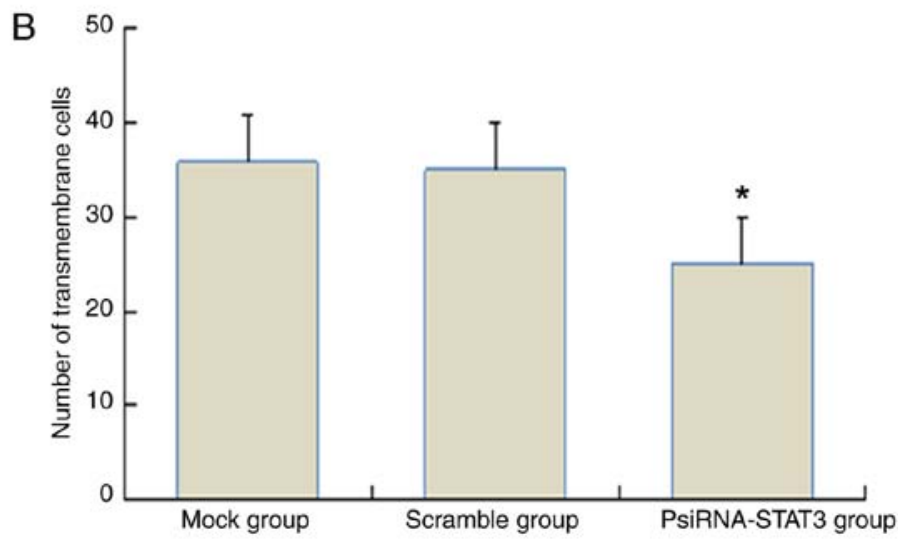

Figure 1. Effect of psiRNA-STAT3 on the invasion ability of C6 glioma cells, assessed by a Transwell assay. (A) Invasion ability of C6 glioma cells (magnification, $\mathrm{x} 20$ ). (B) Number of transmembrane cells in each group. ${ }^{*} \mathrm{P}<0.05$ vs. Scramble and Mock groups. psiRNA, plasmid small interfering RNA; STAT3, signal transducer and activator of transcription-3.

Table I. Effects of psiRNA-STAT3 on the invasion ability of C6 glioma cells.

\begin{tabular}{lc}
\hline Group & No. of transmembrane cells \\
\hline Mock & $35.8 \pm 2.38$ \\
Scramble & $35.0 \pm 3.08^{\mathrm{a}}$ \\
psiRNA-STAT3 & $25.0 \pm 3.74^{\mathrm{b}}$ \\
\hline
\end{tabular}

Data represents the mean \pm standard deviation. ${ }^{\mathrm{a}}>0.05$ vs. Mock group; ${ }^{\mathrm{P}} \mathrm{P}<0.05$, vs. Scramble and Mock groups. psiRNA, plasmid small interfering RNA; STAT3, signal transducer and activator of transcription-3.

Statistical analysis. All data were collected from at least three independent experiments. Results were analyzed using SPSS statistical software (version 13.0; SPSS, Inc., Chicago, IL, USA), and are expressed as the mean \pm standard deviation. Comparisons between groups were examined using Student's t-test. A P-value of $<0.05$ was considered to denote a statistically significant difference.

\section{Results}

Effect of psiRNA-STAT3 on invasion ability of C6 glioma cells. Compared with the Mock and Scramble groups, the number of transmembrane-invading cells was significantly reduced in the psiRNA-STAT3 group $(\mathrm{P}<0.05$; Fig. $1 \mathrm{~A}$ and $\mathrm{B})$. No significant difference in the number of
Table II. Effects of psiRNA-STAT3 on the cell cycle distribution.

\begin{tabular}{llll}
\hline & \multicolumn{3}{c}{ Cell cycle phase } \\
\cline { 2 - 4 } Group & G0/G1 & \multicolumn{1}{c}{$\mathrm{S}$} & $\mathrm{G} 2 / \mathrm{M}$ \\
\hline Mock & $61.55 \pm 0.51$ & $29.90 \pm 1.9$ & $8.51 \pm 1.54$ \\
Scramble & $61.89 \pm 0.98$ & $30.62 \pm 0.93^{\mathrm{a}}$ & $7.48 \pm 1.22$ \\
psiRNA-STAT3 & $62.64 \pm 1.5$ & $25.73 \pm 1.87^{\mathrm{b}}$ & $9.60 \pm 0.74$ \\
\hline
\end{tabular}

Data are presented as the mean \pm standard deviation. ${ }^{\mathrm{a}} \mathrm{P}>0.05$ vs. Mock group; ${ }^{b} \mathrm{P}<0.05$, vs. Scramble and Mock groups. psiRNA, plasmid small interfering RNA; STAT3, signal transducer and activator of transcription-3.

transmembrane cells was observed between the Scramble and Mock groups $(\mathrm{P}>0.05)$, indicating that psiRNA-STAT3 transfection had an inhibitory effect on the invasion ability of C6 glioma cells (Table I, and Fig. 1A and B).

Effect of psiRNA-STAT3 on the cell cycle distribution. Flow cytometric analysis revealed that the proportion of cells in $\mathrm{S}$ phase was markedly decreased in the psiRNA-STAT3 group compared with the Mock and Scramble groups. Furthermore, there was no significant difference among the three groups in other cell cycle phases (Table II and Fig. 2).

Effect of psiRNA-STAT3 on the apoptosis rate. As shown in Fig. 3A and B, transfection with psiRNA-STAT3 clearly promoted the apoptosis of $\mathrm{C} 6$ glioma cells. The cell apoptosis 

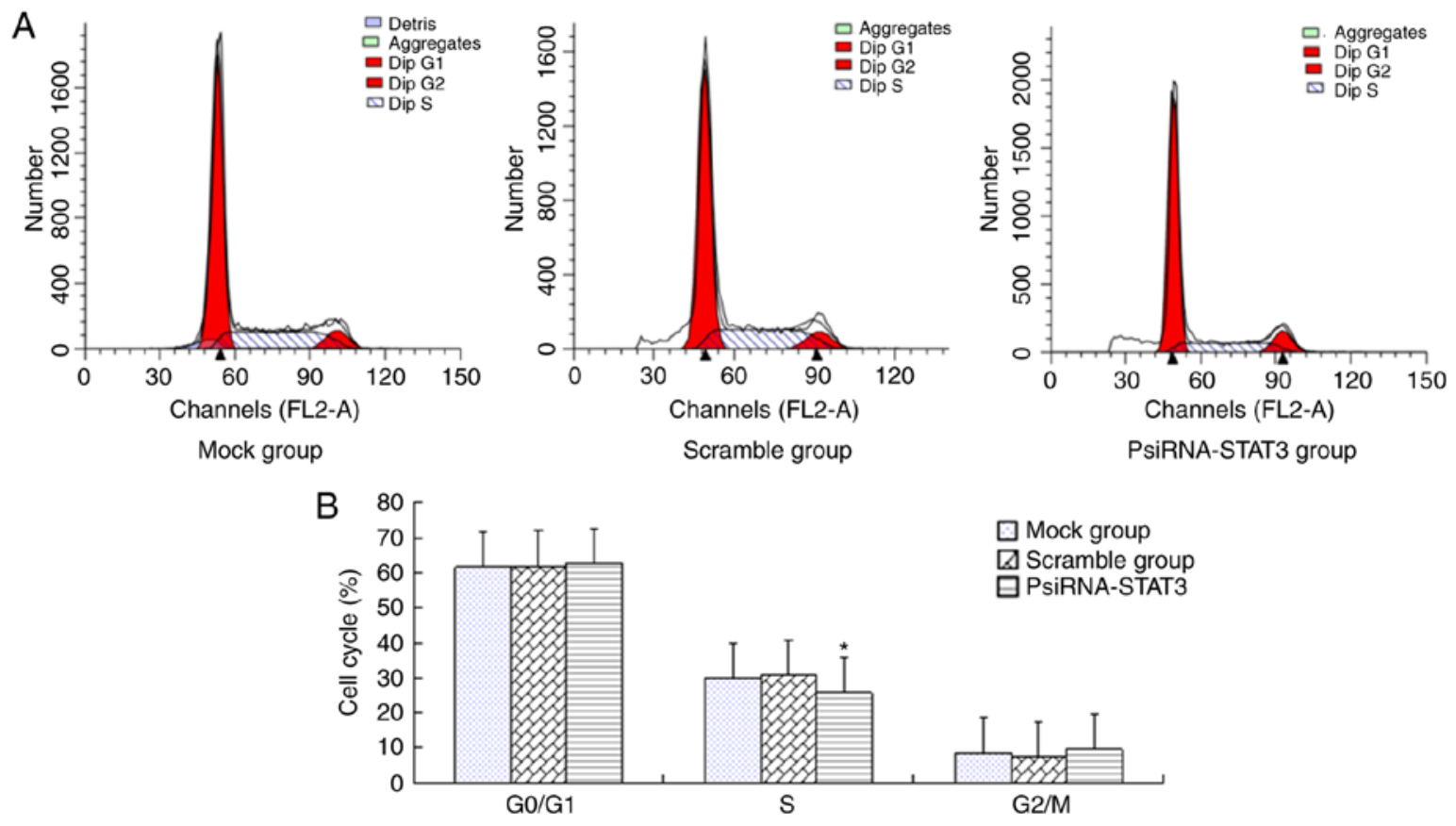

Figure 2. Effects of psiRNA-STAT3 on the cell cycle distribution. (A) Flow cytometry results, and (B) percentage of cells at each cell cycle phase. ${ }^{*} \mathrm{P}<0.05$ vs Scramble and Mock groups. psiRNA, plasmid small interfering RNA; STAT3, signal transducer and activator of transcription-3.

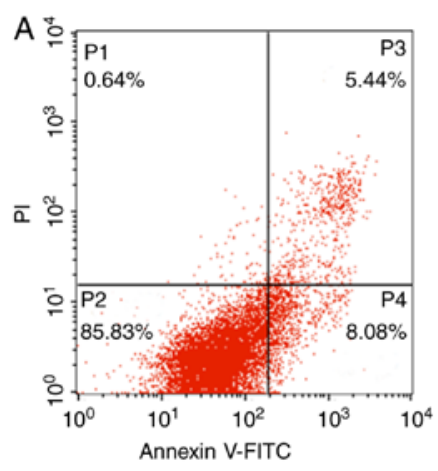

Mock group

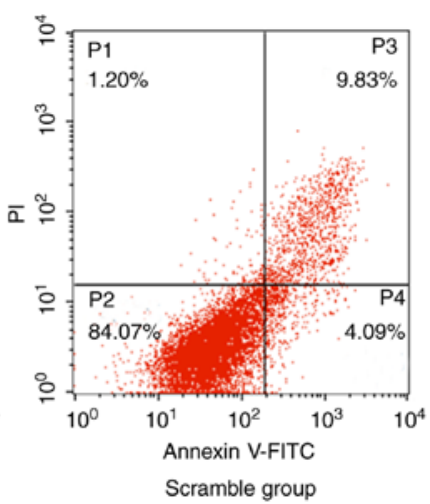

Scramble group

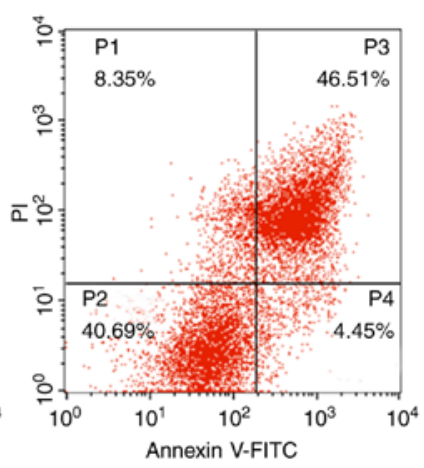

PSIRNA-STAT3 group

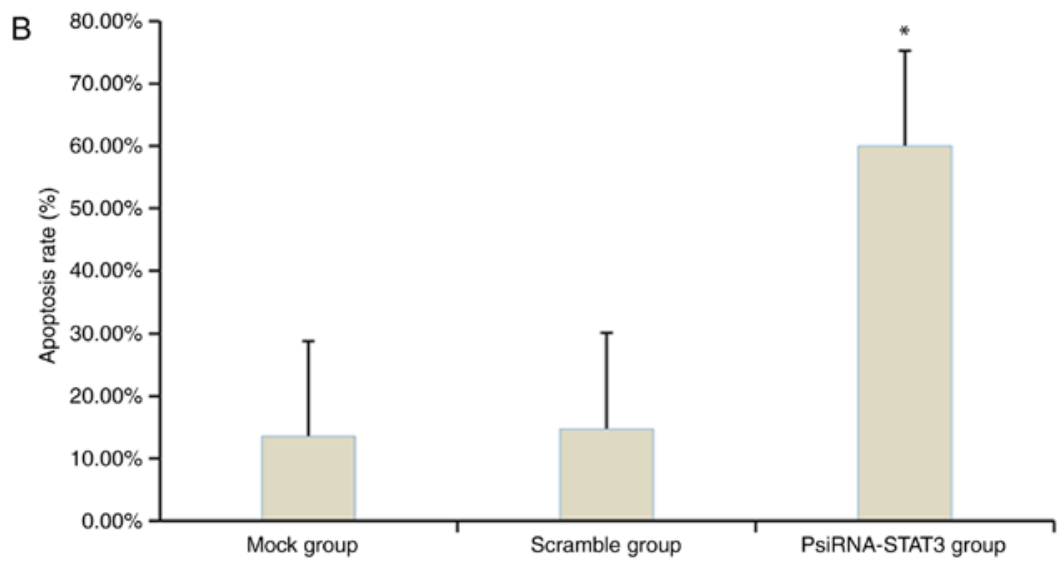

Figure 3. Effect of psiRNA-STAT3 on the apoptosis rate of C6 glioma cells, examined by flow cytometry. (A) Flow cytometry results, and (B) quantified apoptosis rate in each group. Quadrant P1 represents dead cells, quadrant P2 represents non-apoptotic healthy cells, quadrant P3 represents late apoptotic cells, quadrant P4 represents early apoptotic cells. " $\mathrm{P}<0.05$ vs. Mock and Scramble groups. psiRNA, plasmid small interfering RNA; STAT3, signal transducer and activator of transcription-3.

rate was markedly increased in the psiRNA-STAT3 group compared with the Mock and Scramble groups $(\mathrm{P}<0.01)$, whereas no significant difference was detected between the
Mock and Scramble groups. In addition, the late apoptosis rate was 5.44, 9.83 and $46.51 \%$ in the Mock, Scramble and psiRNA-STAT3 groups, respectively. 
Table III. Effect of psiRNA-STAT3 on protein expression levels of cyclin D1, caspase-3 and Bcl-2 in C6 glioma cells.

\begin{tabular}{|c|c|c|c|}
\hline \multirow[b]{2}{*}{ Group } & \multicolumn{3}{|c|}{ Grey level value } \\
\hline & Cyclin D1/ $\beta$-actin & Caspase-3/ $\beta$-actin & Bcl- $2 / \beta$-actin \\
\hline Mock & $0.75 \pm 0.01$ & $1.17 \pm 0.2$ & $0.67 \pm 0.03$ \\
\hline Scramble & $0.71 \pm 0.03^{\mathrm{a}}$ & $0.92 \pm 0.00^{\mathrm{a}}$ & $0.63 \pm 0.03^{\mathrm{a}}$ \\
\hline psiRNA-STAT3 & $0.29 \pm 0.02^{\mathrm{b}}$ & $0.59 \pm 0.08^{b}$ & $0.43 \pm 0.01^{\mathrm{b}}$ \\
\hline
\end{tabular}

Data are expressed as the mean \pm standard deviation. ${ }^{a} \mathrm{P}<0.05$ vs. psiRNA-STAT3 group. ${ }^{\text {b }}<0.05$ vs. Mock group. psiRNA, plasmid small interfering RNA; STAT3, signal transducer and activator of transcription-3; Bcl-2, B-cell lymphoma-2.

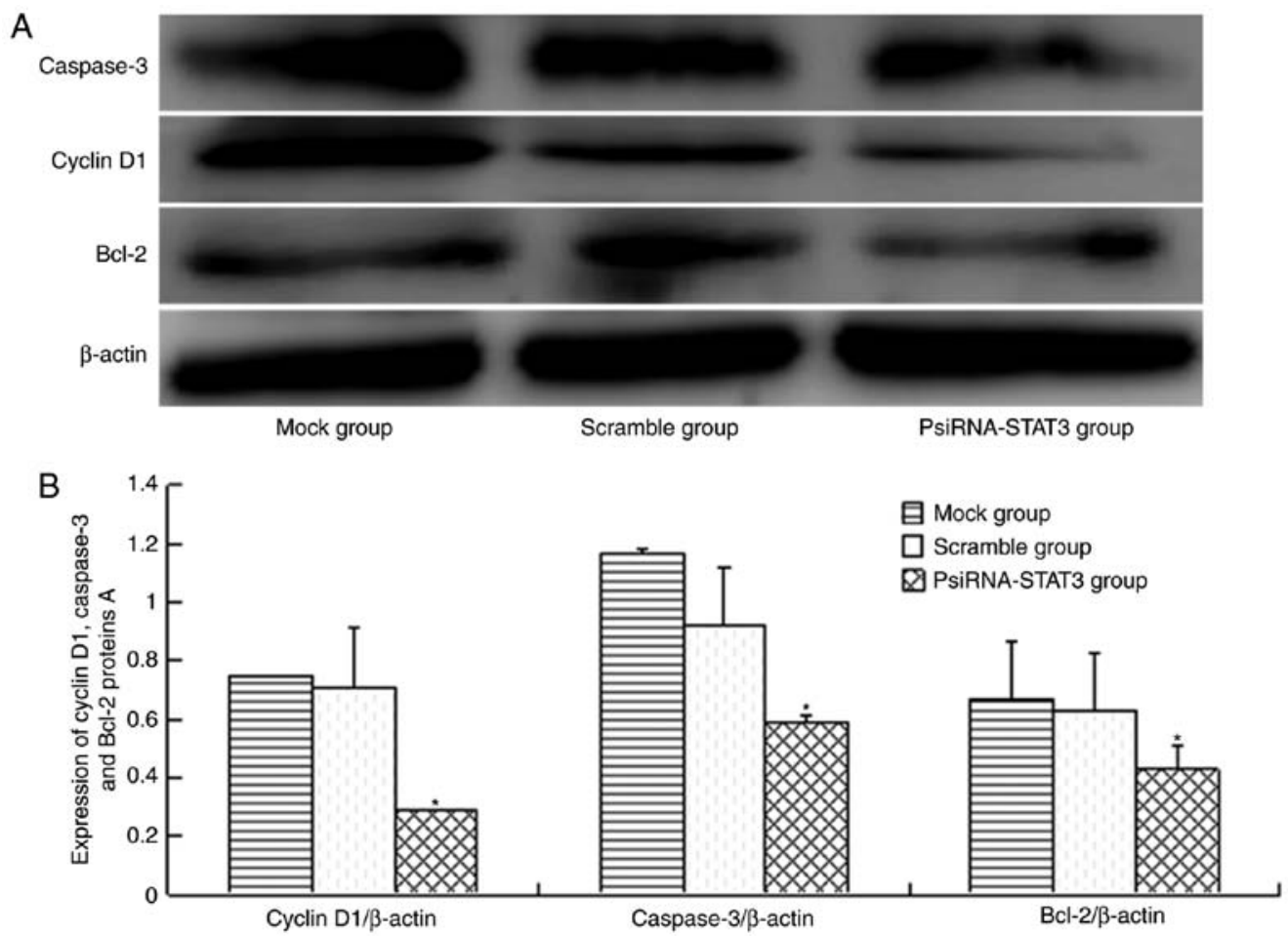

Figure 4. Effects of psiRNA-STAT3 on protein expression levels of cyclin D1, caspase-3, and Bcl-2 in C6 glioma cells. (A) Western blotting results, and (B) quantified levels of protein expression. " $\mathrm{P}<0.05$ vs. psiRNA-STAT3 group. psiRNA, plasmid small interfering RNA; STAT3, signal transducer and activator of transcription-3; Bcl-2, B-cell lymphoma-2.

Effect of psiRNA-STAT3 on protein expression levels of cyclin D1, caspase-3 and Bcl-2. The expression of cyclin D1, caspase-3 and Bcl-2 was relatively high in the Mock and Scramble groups. However, the expression of cyclin D1, caspase-3 and Bcl-2 proteins decreased significantly following transfection with psiRNA-STAT3 $(\mathrm{P}<0.05)$. By contrast, there was no significant difference in the expression of these proteins between the Mock and Scramble groups $(\mathrm{P}>0.05)$. These findings suggest that psiRNA-STAT3 promoted the apoptosis of tumor cells by decreasing the expression levels of cyclin D1, caspase-3 and Bcl-2 proteins (Table III, and Fig. 4A and B).

\section{Discussion}

STAT3 is a member of the STAT family of transcription factors, which can be activated by different cytokines and growth factors. It has been reported that STAT3 has dual functions as signal transducers and transcriptional regulators, and enter the nucleus to affect gene transcription subsequent to receiving an external signal stimulus (16-18). STAT3 is of particular interest due to its close association with tumors, and there is mounting evidence that activated STAT3 serves a key role in malignant transformation $(19,20)$. In view of this, STAT3 is considered to be a novel target for tumor treatment; therefore, specific targeting of STAT3 signaling may have therapeutic potential for malignant gliomas (21).

It is well known that tumor invasion and metastasis are important biological characteristics of malignant tumors. In the present study, the effect of psiRNA-STAT3 transfection on the migration and invasion of C6 glioma cells was investigated. The data demonstrated a significant decrease in the number of transmembrane cells in the psiRNA-STAT3 group $(\mathrm{P}<0.05)$ when compared with the Mock and Scramble groups, 
suggesting that psiRNA-STAT3 inhibited the migration and invasion abilities of C6 glioma cells.

Cyclin D1 is regarded as a type of proto-oncogene (22) whose overexpression leads to carcinogenesis through the lack of control of cell cycle regulation, notably, the transition between G1/S phase and G2/M phase (23). Cyclin D1 overexpression shortens the G1 phase, leading to advanced $S$ phase and hyperplasia $(24,25)$. The data of the present study indicated that psiRNA-STAT3 altered the cell cycle progression by affecting the regulation of cyclin D1 protein expression in glioma cells. psiRNA-STAT3 treatment increased the number of cells arrested at G0/G1 phase, leading to a significant decrease in the number of cells in $\mathrm{S}$ phase as compared with the Mock and Scramble groups $(\mathrm{P}<0.05)$ and thus inhibiting cell proliferation. Acridine orange/ethidium bromide staining in our previous research also revealed that the proportion of cells in late apoptosis was higher in the psiRNA-STAT3 group in comparison with that in the Mock and Scramble groups (16). Furthermore, western blotting data in the present study further revealed that cyclin D1 protein expression was significantly decreased in the psiRNA-STAT3 group as compared with that in the Mock and Scramble groups $(\mathrm{P}<0.05)$.

Caspases are cysteine-containing proteolytic enzymes that are closely associated with apoptosis in eukaryotic cells, and are involved in cytokine maturation, cell growth and differentiation. The signal transduction mechanisms of apoptosis include the death receptor, mitochondrial and endoplasmic reticulum pathways. Signal transduction is initiated with the activation of caspases, and the final outcome is the cleavage of protein substrates and the breakdown of cells. Under normal conditions, caspases exist as inactive zymogens. When caspases are activated by apoptotic signals, upstream caspases sequentially activate downstream caspases in a cascade reaction and transmit apoptotic signals (26). Caspase-3 is the most important inducer of apoptosis in the caspase family, and the activation of caspase- 3 can directly lead to apoptosis (27). In the present study, the results of western blotting confirmed that there was no significant difference in caspase-3 expression between the Scramble and the Mock groups, whereas caspase-3 expression was significantly decreased in the psi-STAT3 group as compared with the Mock and Scramble groups $(\mathrm{P}<0.05)$.

$\mathrm{Bcl}-2$ has previously been reported to indirectly promote the occurrence and development of tumors by inhibiting glioma C6 cell apoptosis (28). Bcl-2 is one of the most important regulators of apoptosis among the Bcl-2 family, and its activation can lead to the release of cytochrome $c$ via the mitochondrial pathway. This influences the activation of caspase-3, which sets off a downstream cascade reaction. In the present study, a significant decrease in the expression of the anti-apoptotic protein $\mathrm{Bcl}-2$ was also observed in the psiRNA-STAT3 group in comparison with its expression in the Mock and Scramble groups $(\mathrm{P}<0.05)$. Taken together, the present study revealed that the expression levels of cyclin D1, Bcl-2 and caspase-3 were significantly reduced in the psiRNA-STAT3 group, indicating that STAT3 silencing promoted glioma cell apoptosis.

In conclusion, the results reported in the present study revealed that psiRNA-STAT3 promoted the apoptosis of glioma cells by decreasing the expression levels of cyclin D1, Bcl-2 and caspase-3. Furthermore, the expression of caspase-3 was positively associated with that of Bcl-2, and psiRNA-STAT3's mechanism of action may inhibit caspase-3 activity. This would result in a decrease in Bcl-2 expression, leading to apoptosis and inhibiting the development of glioma. These findings provide a new theoretical basis for the clinical treatment of glioma.

\section{Acknowledgements}

Not applicable.

\section{Funding}

This study was supported by the Precision Medical Science and Technology Innovation Center for Nervous System Diseases of Jilin Province (grant no. 20170623006TC, awarded to Jiajun Chen).

\section{Availability of data and materials}

The datasets used and/or analyzed during the current study are available from the corresponding author on reasonable request.

\section{Authors' contributions}

QZ designed and supervised the study. JC designed the study and wrote the paper. XZ, YZ, JL and FL reviewed the manuscript, analyzed the data and performed the experiments. All authors read and approved the final manuscript.

\section{Ethics approval and consent to participate}

Not applicable.

\section{Patient consent for publication}

Not applicable.

\section{Competing interests}

The authors declare that they have no competing interests.

\section{References}

1. Roci E, Cakani B, Brace G, Bushati T, Rroji A, Petrela M, Kaloshi G: Platinum-based chemotherapy in recurrent high-grade glioma patients: Retrospective study. Med Arch 68: 140-143, 2014.

2. Feng B, Hu P, Lu SJ, Chen JB and Ge RL: Increased argonaute 2 expression in gliomas and its association with tumor progression and poor prognosis. Asian Pac J Cancer Prev 15: 4079-4083, 2014.

3. Davis ME and Stoiber AM: Glioblastoma multiforme: Enhancing survival and quality of life. Clin J Oncol Nurs 15: 291-297, 2011.

4. McNamara MG and Mason WP: Antiangiogenic therapies in glioblastoma multiforme. Expert Rev Anticancer Ther 12: 643-654, 2012.

5. Vaupel P: Hypoxia and aggressive tumor phenotype: Implications for therapy and prognosis. Oncologist 13: 21-26, 2008.

6. Mazure NM and Pouysségur J: Hypoxia-induced autophagy: Cell death or cell survival? Curr Opin Cell Biol 22: 177-180, 2010 
7. Sayegh ET, Kaur G, Bloch O and Parsa AT: Systematic review of protein biomarkers of invasive behavior in glioblastoma. Mol Neurobiol 49: 1212-1244, 2014.

8. Zhou M, Wang H, Zhou K, Luo X, Pan X, Shi B, Jiang H, Zhang J, Li K, Wang HM, et al: A novel EGFR isoform confers increased invasiveness to cancer cells. Cancer Res 73: 7056-7067, 2013.

9. Coussens LM, Fingleton B and Matrisian LM: Matrix metalloproteinase inhibitors and cancer: Trials and tribulations. Science 295: 2387-2392, 2002.

10. Friedl P and Wolf K: Plasticity of cell migration: A multiscale tuning model. J Cell Biol 188: 11-19, 2010.

11. Hanahan D and Weinberg RA: Hallmarks of cancer: The next generation. Cell 144: 646-674, 2011.

12. Hong SS, Lee H and Kim KW: HIF-1alpha: A valid therapeutic target for tumor therapy. Cancer Res Treat 36: 343-353, 2004.

13. Morioka Y, Monypenny J, Matsuzaki T, Shi S, Alexander DB, Kitayama $\mathrm{H}$ and Noda M: The membrane-anchored metalloproteinase regulator RECK stabilizes focal adhesions and anterior-posterior polarity in fibroblasts. Oncogene 28: 1454-1464, 2009.

14. Doucette TA, Kong LY, Yang Y, Ferguson SD, Yang J, Wei J, Qiao W, Fuller GN, Bhat KP, Aldape K, et al: Signal transducer and activator of transcription 3 promotes angiogenesis and drives malignant progression in glioma. Neuro Oncol 14: 1136-1145, 2012 .

15. Zhou XY, Tian L, Zhou Y, Dai L, Liu S and Zhou QW: The effect of Lipofectamine 2000 plasmid on glioma C6 cells. Zhongguo Laonianxue Zazhi 36: 3404-3406, 2016.

16. Yuan J, Zhang F and Niu R: Multiple regulation pathways and pivotal biological functions of STAT3 in cancer. Sci Rep 5: 17663, 2015.

17. Yu H, Lee H, Herrmann A, Buettner R and Jove R: Revisiting STAT3 signalling in cancer: New and unexpected biological functions. Nat Rev Cancer 14: 736-746, 2014.

18. Geiger JL, Grandis JR and Bauman JE: The STAT3 pathway as a therapeutic target in head and neck cancer: Barriers and innovations. Oral Oncol 56: 84-92, 2016.

19. Tanaka S, Suto A, Iwamoto T, Kashiwakuma D, Kagami S, Suzuki K, Takatori H, Tamachi T, Hirose K, Onodera A, et al: Sox 5 and c-Maf cooperatively induce Th17 cell differentiation via ROR $\gamma$ t induction as downstream targets of Stat3. J Exp Med 211: 1857-1874, 2014.
20. Yang YC, Huang YT, Hsieh CW, Yang PM and Wung BS: Carbon monoxide induces heme oxygenase-1 to modulate STAT3 activation in endothelial cells via S-glutathionylation. PLoS One 9: e100677, 2014.

21. Doucette TA, Kong LY, Yang Y, Ferguson SD, Yang J, Wei J, Qiao W, Fuller GN, Bhat KP, Aldape K, Priebe W, Bogler O, Heimberger AB, Rao G. Signal transducer and activator of transcription-3 promotes angiogenesis and drives malignant progression in glioma. Neuro Oncol 14(9):1136-45, 2012.

22. Inaba T, Matsushime H, Valentine M, Roussel MF, Sherr CJ and Look AT: Genomic organization, chromosomal localization, and independent expression of human cyclin D genes. Genomics 13: 565-574, 1992.

23. Vansaun MN: Molecular pathways: Adiponectin and leptin signaling in cancer. Clin Cancer Res 19: 1926-1932, 2013.

24. Fox MM, Phoenix KN, Kopsiaftis SG and Claffey KP: AMP-activated protein kinase $\alpha 2$ isoform suppression in primary breast cancer alters ampk growth control and apoptotic signaling. Genes Cancer 4: 3-14, 2013.

25. Zhuang Y, Chan DK, Haugrud AB and Miskimins WK Mechanisms by which low glucose enhances the cytotoxicity of metformin to cancer cells both in vitro and in vivo. PLoS One 9: e108444, 2014

26. Yihua Chen YH, Zhang HJ and Nan FJ: Progress in the research of Caspases inhibitor. Shengwu Jishu 18: 248-253, 2006.

27. Sikdar S, Mukherjee A, Ghosh S and Khuda-Bukhsh AR: Condurango glycoside-rich components stimulate DNA damage-induced cell cycle arrest and ROS-mediated caspase-3 dependent apoptosis through inhibition of cell-proliferation in lung cancer, in vitro and in vivo. Environ Toxicol Pharmacol 37: 300-314, 2014.

28. Nahta R and Esteva FJ: Bcl-2 antisense oligonucleotides: A potential novel strategy for the treatment of breast cancer. Semin Oncol 30: 143-149, 2003. 